PETROVIETNAM JOURNAL

Volume 10/2021, p. 4 - 16

ISSN 2615-9902

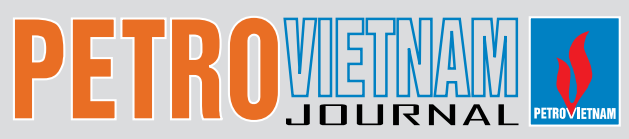

\title{
SOME RESULTS OF SEISMIC TRAVEL-TIME REFLECTION TOMOGRAPHY STUDY
}

\author{
Pham The Hoang Ha, Doan Huy Hien, Ta Quang Minh, Mai Thi Lua, Nguyen Hoang Anh \\ Vietnam Petroleum Institute \\ Email: hapth@vpi.pvn.vn \\ https://doi.org/10.47800/PVJ.2021.10-01
}

\section{Summary}

Velocity model is essential for seismic data processing as it plays an important role in migration processes as well as time depth conversion. There are several techniques to reach that goal, among which tomographic inversion is an efficient one. As an upgrade version of handpicked velocity analysis, the tomography technique is based on the reflection ray tracing and conjugate gradient method to estimate an optimum velocity model and can create an initial high quality model for other intensive imaging and modelling module such as reverse-time migration (RTM) and full-waveform inversion (FWI). For the mentioned benefit, we develop a seismic travel-time reflection tomography (SeisT) module to study the accuracy of the approach along with building the technical capability in seismic processing. The accuracy of the module has been tested by both synthetic and real seismic field data; the efficiency and the accuracy of the model have been proven in terms of development method as well as field data application.

Key words: Seismic signal processing, seismic reflection, ray tracing, velocity modelling, seismic tomography, tomographic inversion.

\section{Introduction}

Velocity model is one of the most important features extracted from the seismic reflection data as it has been used for many purposes such as depth imaging, timedepth conversion and geomechanical model building. The resolution of velocity model building is highly dependent on the complexity of the method to be used and the resolution of the initial model and seismic data. For example, the velocity analysis is the simplest and fastest way to create the velocity model, however, the resolution of this method is poor in both lateral and time directions as the distance between two picking common depth points (CDP) is much higher than the one between two initial CDP intervals. Similarly, within a CDP semblance spectrum for velocity picking, the picking time interval is much higher than the time sampling rate. Full waveform inversion (FWI), in the reverse way, gives the velocity model the highest resolution and accuracy. However, the computational

Date of receipt: 19/3/2021. Date of review and editing: 19/3 - 23/4/2021.

Date of approval: 7/10/2021. cost is too high to be used widely in the seismic reflection industry. To reach the balance between the accuracy and computational cost, the seismic reflection tomography (SeisT) approach is an alternative method for getting the velocity model for migration purposes and to be used as the initial model for FWI problem, which produces a higher resolution than SeisT.

Tomography is an inversion process that provides a tool for velocity estimations from multichannel seismic reflection data. Tomography can be performed either in the prior-migration domain or in the post-migration domain. In each of these domains, we can access two types of information: kinematic (travel time) and dynamic (amplitude and phase) [1]. Thus, we have at least four ways to sort the tomographic inverse problem out. Table 1 gives a summary of the approaches used for velocity estimation.

During this work, we demonstrate a study of raybased reflection travel time tomography applied for synthetic and field velocity models. The workflow of this approach is shown in Figure 1. There are several reasons why we chose this kind of approach for studying velocity 
Table 1. Types and domains of tomography for velocity estimation [1]

\begin{tabular}{|l|l|l|}
\hline & \multicolumn{1}{|c|}{ Prior-migration domain } & \multicolumn{1}{c|}{ Post-migration domain } \\
\hline $\begin{array}{l}\text { Ray-based } \\
\text { (kinematic) }\end{array}$ & $\begin{array}{l}\text { Reflection travel-time tomography } \\
\text { Cross-well transmission tomography } \\
\text { Refraction tomography }\end{array}$ & $\begin{array}{l}\text { Pre-stack time migration tomography } \\
\text { Pre-stack depth migration tomography }\end{array}$ \\
\hline $\begin{array}{l}\text { Waveform-based } \\
\text { (dynamic) }\end{array}$ & $\begin{array}{l}\text { Full waveform inversion (also known as waveform tomography, wave } \\
\text { equation tomography, and diffraction tomography) }\end{array}$ & $\begin{array}{l}\text { Wave-equation migration velocity analysis (WEM-VA) } \\
\text { Wavepath tomography }\end{array}$ \\
\hline
\end{tabular}

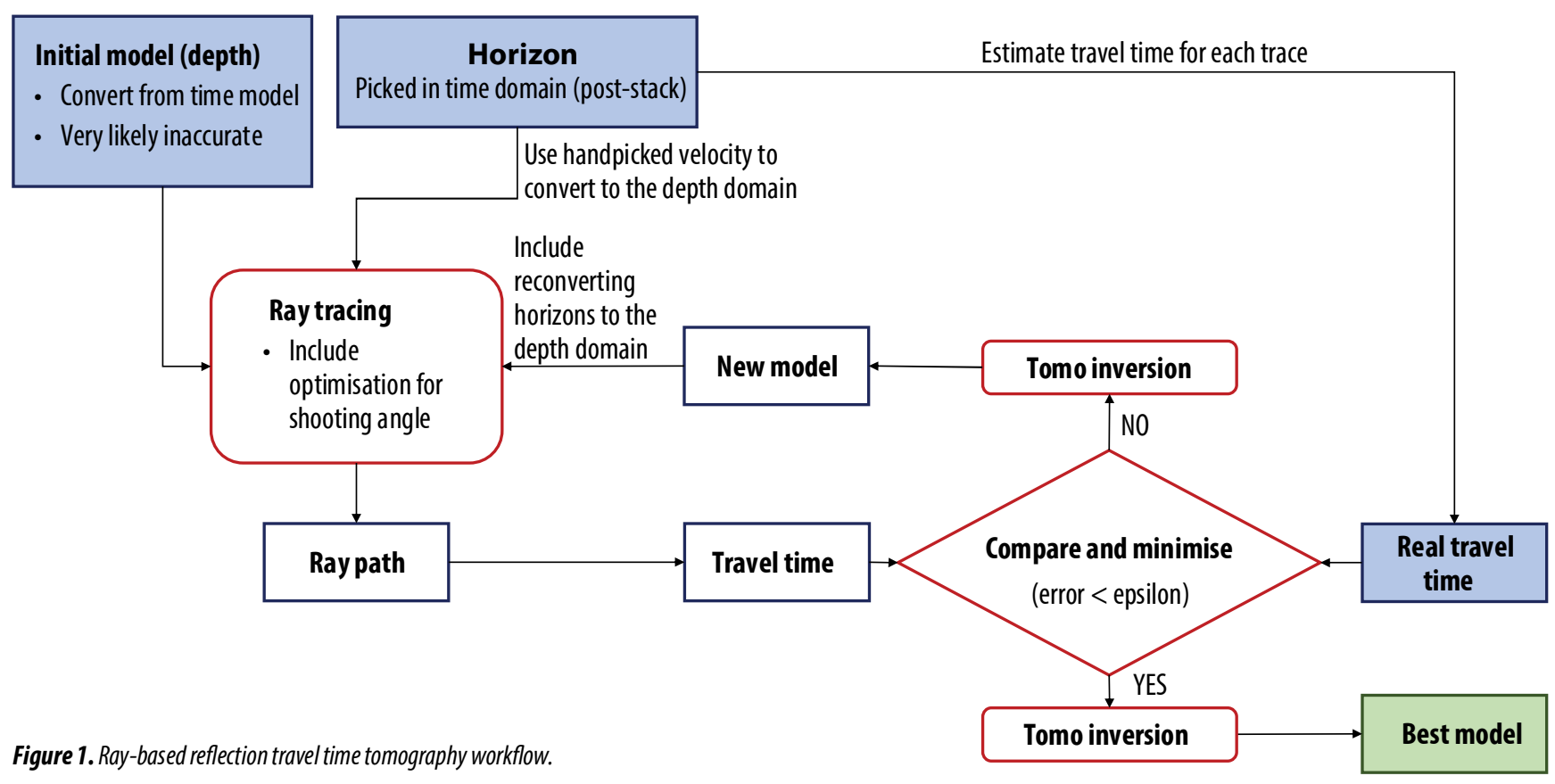

estimation. Firstly, there is more physical information contained in the prior-migration domain than in the postmigration domain, where data has been migrated by using a not-optimised velocity model. Secondly, although waveform-based tomography can create a highly detailed velocity model, it demands a large computational cost. The ray-based method produces a velocity model of less resolution but still has the advantage of "robustness" (good quality), when carefully implemented [2].

\section{Theoretical background}

Like many other geophysical inverse problems, the ray-based reflection travel-time tomography consists of two basic steps:

- Determine a set of seismic reflectors and estimated travel times for various source-receiver positions based on the Eikonal equation's solution.

- Iteratively update the velocity model by minimising the difference between calculated travel time and observation travel time. In this algorithm, the conjugate gradient method is adopted to build a best-fit velocity model by iteratively comparing estimated travel times with the observed travel time for a given set of horizons.

\subsection{Seismic ray tracing formulation and its numerical solution}

In this step, the procedure of estimating travel times is performed by Runge-Kutta ray tracing technique in depth domain. Hence, for real data, all input objects of ray tracing procedure, including starting model and seismic horizons, must be converted from time to depth domain. In case of synthetic data, converting is not required because the velocity model is already in depth domain and all reflectors will be hand-picked on this model.

Ray tracing is a method for estimating the ray path through a media of varying propagation velocity, absorption characteristics, and reflecting surfaces. Under these circumstances, the ray path may bend, change direction, or reflect off surfaces, thus complicating analysis. 
The ray trajectories are found by solving a certain differential equation that can be derived from the wave equation as follows [3]:

$$
\nabla^{2} \psi-\frac{1}{v^{2}(\vec{x})} \frac{\partial^{2} \psi}{\partial t^{2}}=0
$$

Equation 1 has a solution assumed in the form:

$$
\psi(\vec{x}, t)=A(\vec{x}) e^{2 \pi i f(t-T(\vec{x}))}
$$

where $A(x)$ and $T(x)$ are unknown functions describing amplitude and travel time that are expected to vary with position.

By substituting Equation 2 into Equation 1 and considering weak velocity gradients as well as high frequencies regardless of the velocity gradient, we achieve the following results:

$$
\begin{gathered}
{[\vec{\nabla} T]^{2}-\frac{1}{v(\vec{x})^{2}}=0} \\
\frac{A}{2} \nabla^{2} T+\vec{\nabla} A \cdot \vec{\nabla} T=0
\end{gathered}
$$

Equation 4 is called the geometrical spreading equation because its solution can be shown to describe the flow of energy along a ray path.

Equation 3 is the non-linear, partial-differential equation called the eikonal equation. For high frequencies or small velocity variations, a solution to the eikonal equation gives accurate travel times through complex media [3]. Using the method of characteristics, we can transform this equation into a system of first-order ordinary differential equations (ODE) that can be solved by standard numerical methods

$$
\begin{aligned}
& \frac{d \vec{x}}{d t}=v^{2}(\vec{x}) \vec{p} \\
& \frac{d \vec{p}}{d t}=-\frac{\vec{\nabla} v(\vec{x})}{v(x)} .
\end{aligned}
$$

where $\vec{p}$ is slowness vector.

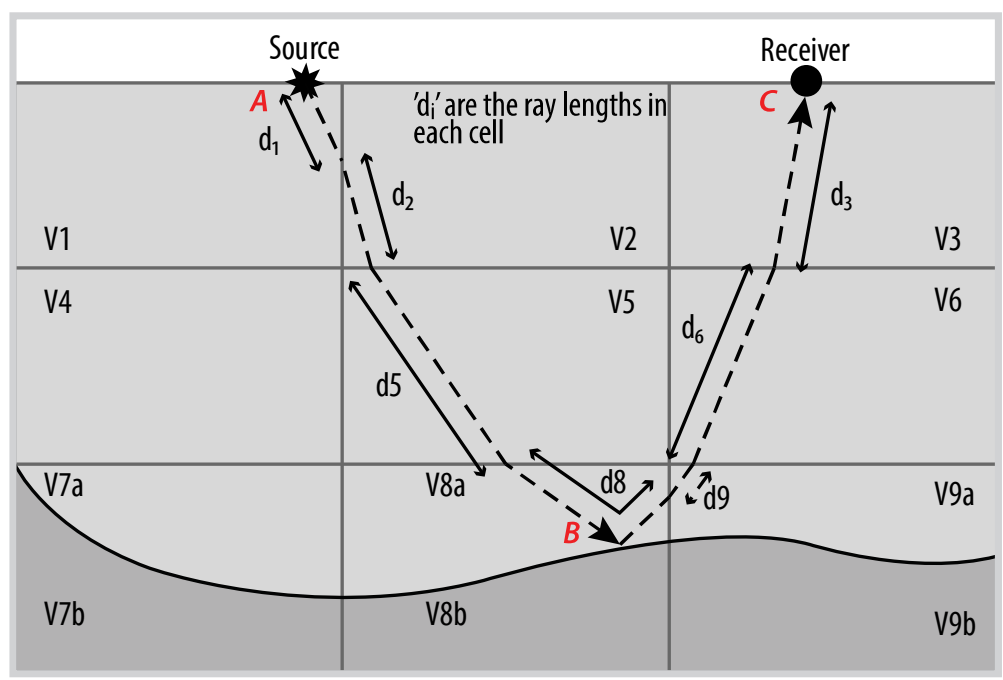

Figure 2. Graphical representation of ray tracing in velocity field [1].
Our study adopts the Runge-Kutta method to solve this ODE system [4].

The time step $\mathrm{dt}$ for solving the ODE system (Equation 5) must be chosen to be consistent with the velocity grid. If $\mathrm{dt}$ is too big, the velocity model will be updated sparsely, because the conjugate gradient method will edit only grids, where rays propagate through. If $\mathrm{dt}$ is too small, the number of tracing steps for a ray to reach its intended destination will be large and this will also lead to heavy computational cost. In our study, we apply an additional linear interpolation to ensure that every grid on the ray path is updated in the iterative procedure.

Our velocity field is discretised into samples for computational simulation. In this field, the ray trajectory is estimated repeatedly after every fixed time interval or step (Figure 2). At the end of each step, the current position of a tracing ray is updated and the values of the velocity and ray parameters at the nearest sample to this position are used to define the next position of the ray.

During simulated seismic acquisition, waves initiate at a shooting point (source) at or near the surface, propagate through the Earth's layers and may reach the receiving point (receiver), also at or near the surface, by reflection, refraction, or both. This complicated process can be described on a velocity model, as mentioned above, by using the RungeKutta ray tracing technique. Figure 1 shows the graphical representation of this model where simulated rays are reflected off the picked horizon and turn back to the surface. A distinctive feature of reflection tomography is that the ray will be reflected off a chosen horizon before arriving at the surface receiver. The calculation of the slowness vector of a reflected ray is demonstrated in Figure 3, where $\vec{p}_{1}$ is the incident ray's slowness and $\vec{p}_{2}$ is the reflected ray's slowness vectors. In the co-ordinate system Ogh at the reflection point on the horizon, the tangent opponent of the slowness vector is unchanged, while the normal opponent is changed in sign. Thus, components of the reflected slowness vector 
are computed from those of the given incident slowness vector as follows:

$$
\begin{aligned}
& p g_{2}=p g_{1} \\
& p h_{2}=-p h_{1}
\end{aligned}
$$

where $\mathrm{pg}_{1,2}$ and $\mathrm{ph}_{1,2}$ denote as the incident ray and reflected ray slowness, respectively.

Additionally, the reflected rays as shown in Figure 2 could also travel in many other ways such as the turning ray that may never reach the intended reflector as in Figure 8. This is due to the total internal reflection at the shallower reflector (the incident angle is greater than the critical angle). Though the turning ray is also very useful for near surface tomography (such as building the velocity model for seismic land survey), it is not the target of this study.

A crucial issue for ray tracing is that for a particular pair of surface source-and-receiver, how do we determine the shooting angle for the ray from the source so that it could reach the intended receiver's location? If we shoot the ray by an arbitrary angle, it is likely not to reach the target receiver. Our solution for this issue is to use shooting angles determined by several methods. A simple way to try is to use a basic geometrical calculation as shown in Figure 4. Given the location of $S$ and $R$ (source and receiver) and the dip $\beta$ of the reflector (near the location of CDP), the shooting angle can be computed by the formula:

$$
\alpha=\gamma-\beta=\tan ^{-1}\left(\frac{d}{2 h}\right)-\beta
$$

where $d$ is the source-receiver offset and his the depth of the projection point of the midpoint on the horizon.

As this ray tracing simulation was not utilised well for estimating an optimum shooting angle, the geometrically calculated value was used as an initial guess to optimise the procedure. Several optimisation algorithms such as bisection search, Gauss-Newton algorithm and the steepest descent method [4] have been tested, and we find out that the latter usually gives the best angle estimates for an acceptable number of iterations. In some exceptional cases when the steepest descent does not work properly due to the local minimum convergence, the bisection search will be employed to reach the nearest possible point to the receiver position.

For far-offset-receivers on the same source record, we can apply the least-squares regression [4] to the set of near-offset shooting angles (already calculated/optimised) to arrive at a more accurate starting value for the steepest descent calculation of the current shooting angle. By this way, the number of steepest descent iterations will be much less than that in the case of using the geometrical initial angle estimation. In Table 2, we summarised the way to find out the optimum.

A particular notice during our experiments to improve optimisation convergence was that a smoothing filter would need to be applied to the velocity model and all horizons. Moreover, the speed and accuracy of the angle finding process are also enhanced significantly.

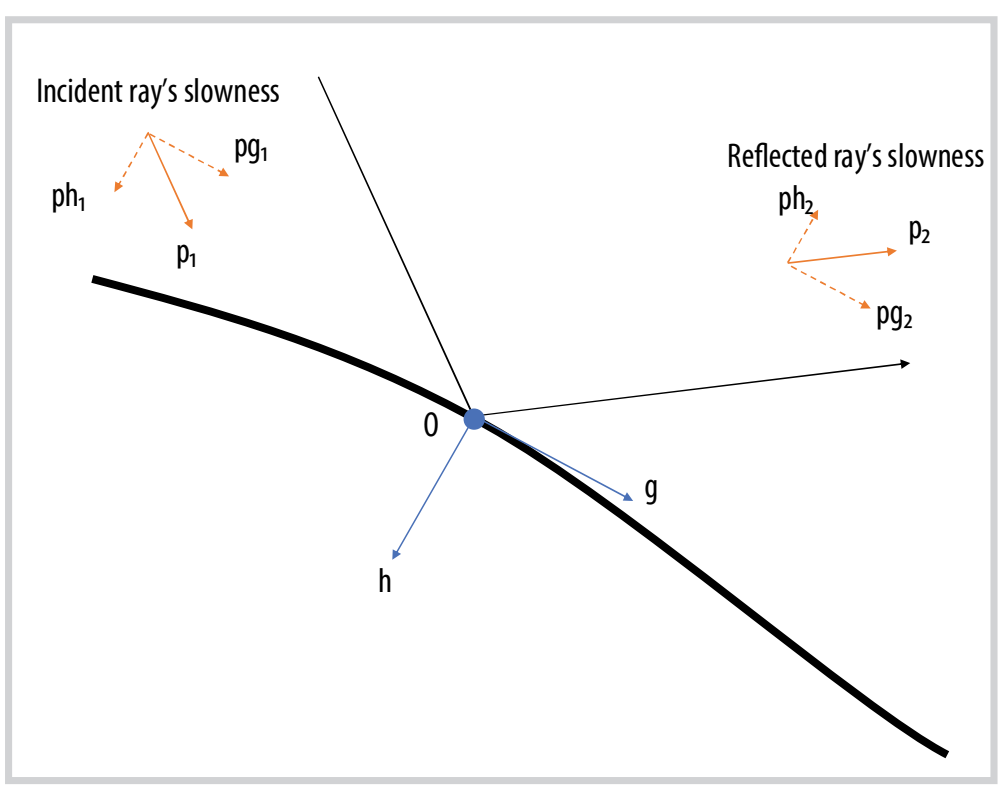

Figure 3. Recalculation of slowness vector at the point of reflection.

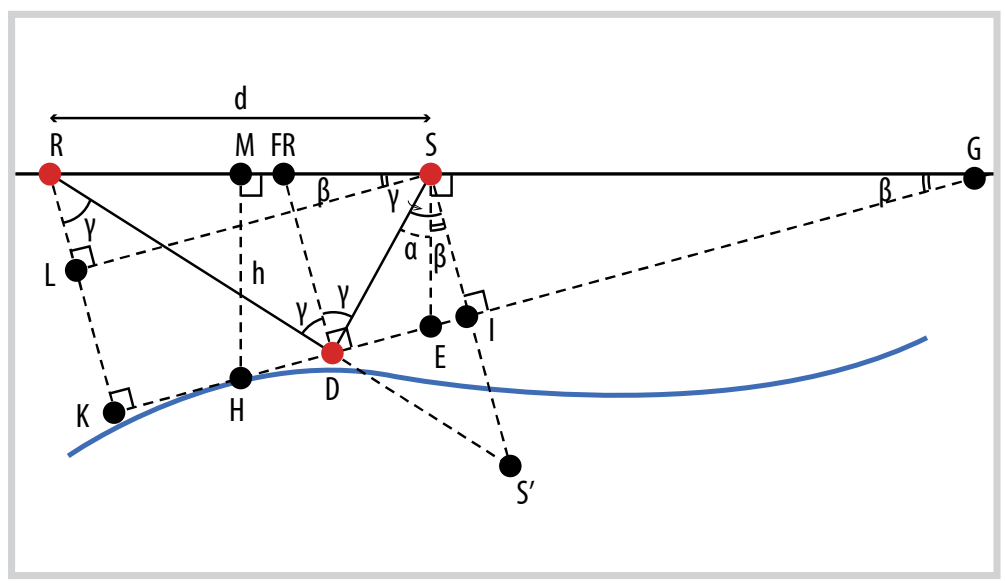

Figure 4. Geometrical representation of angle finding problem. 
Table 2. Number of iterations of angle estimation procedure with acceptable error $\pm 3 \mathrm{~m}$ around receiver's location

\begin{tabular}{|c|c|c|c|c|c|c|c|}
\hline & & \multicolumn{5}{|c|}{ Source's location (m) } & \multirow{2}{*}{$\begin{array}{l}\text { Method to calculate initial guess for } \\
\text { the steepest descent optimisation }\end{array}$} \\
\hline & & 0 & 1,000 & 2,000 & 3,000 & 4,000 & \\
\hline \multirow{10}{*}{ Offset (m) } & 125 & 4 & 2 & 4 & 3 & 2 & \multirow{2}{*}{ Geometrical } \\
\hline & 137.5 & 2 & 3 & 3 & 2 & 2 & \\
\hline & 150 & 0 & 1 & 2 & 1 & 0 & \multirow{8}{*}{ Least-squares regression } \\
\hline & 162.5 & 0 & 1 & 1 & 1 & 0 & \\
\hline & 175 & 0 & 0 & 0 & 1 & 1 & \\
\hline & 187.5 & 0 & 1 & 2 & 1 & 1 & \\
\hline & 200 & 0 & 1 & 1 & 0 & 1 & \\
\hline & 212.5 & 0 & 1 & 1 & 1 & 1 & \\
\hline & 225 & 0 & 1 & 1 & 1 & 0 & \\
\hline & 237.5 & 1 & 1 & 1 & 1 & 1 & \\
\hline
\end{tabular}

\subsection{Inversion problem}

If the velocity model is very deviated from the actual one, the errors between estimated travel times by the simulated ray tracing and their true values will be large. We thus need to minimise these errors using the optimisation routine by gradually adjusting the velocity model toward that goal. By this way, the estimated velocity model will gradually approach the actual one.

Consider the velocity model described earlier in Figure 2, which is divided into nine constant velocity cells, and a single ray reflecting off a horizon at point $B$, for a source at $A$ and a receiver at $C$. The arrival time $T_{A B C}$ for the ray path $A B C$ is given by Equation 7 as:

$T_{A B C}=\frac{d_{1}}{v_{1}}+\frac{d_{2}}{v_{2}}+\frac{d_{5}}{v_{5}}+\frac{d_{8}}{v_{8 a}}+\frac{d_{9}}{v_{9 a}}+\frac{d_{6}}{v_{6}}+\frac{d_{3}}{v_{3}}(8)$

Using many ray paths traversing the cells in the model, we can obtain a set of simultaneous equations (with measured travel time $\mathrm{T}$ and the unknowns are elements $v_{i}$ of the velocity field). The task of solving those simultaneous equations (known as the tomography inversion) will result in the determination of the velocity distribution along such ray paths.

We have many arrival time measurements for a given sub-surface reflector element. Consider the five ray paths gathering shown in Figure 5, and the associated arrival times along the moveout trajectory (Figure 6). The travel time expression for these five ray paths can be written as:

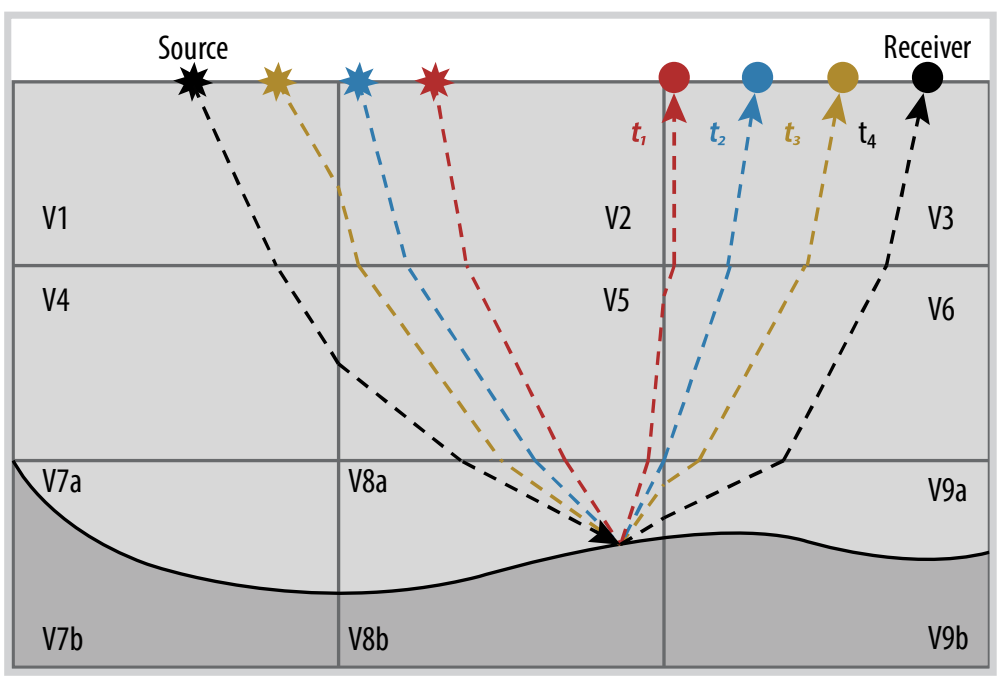

Figure 5. Input gathering in nine cell model $(j=1,9)[1]$.

$$
t_{i}=\sum_{j=1}^{N} \frac{d_{i j}}{v_{j}}=\sum_{j=1}^{N} d_{i j} s_{j}
$$

Or, in matrix notation:

$$
T=D S
$$

where $t_{i}$ is the total travel time along the $i^{\text {th }}$ ray path;

$d_{i j}$ is the path length in the $j^{\text {th }}$ cell of the velocity model for $i^{\text {th }}$ the ray;

$v_{j}$ is the velocity in the $j^{\text {th }}$ cell;

$\mathrm{s}_{\mathrm{j}}$ is the slowness in the $\mathrm{j}^{\text {th }}$ cell, where we have $\mathrm{N}$ cells in the model (in this example, $\mathrm{N}=9$ ).

In Equation 10, $\mathrm{T}$ is a vector of two-way travel time measured for sound waves emanating from a source, propagating through the earth and reflecting off a horizon, then returning to an individual receiver. $D$ is a matrix of path lengths that the ray paths have in each cell of the velocity model (Figure 2) [1]. The matrix D is determined in step 1 using ray tracing technique. $S$ is a vector of velocity model and will be estimated numerically. 


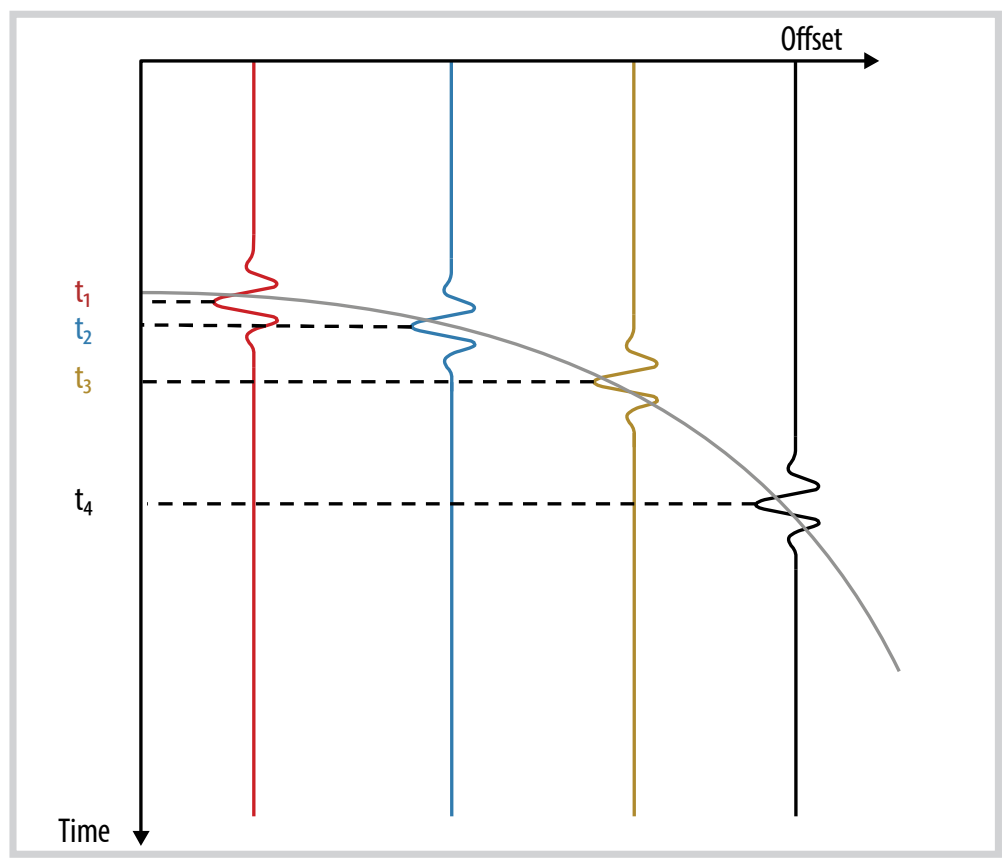

Figure 6. Moveout trajectory for a reflector: an autopicker will determine the t values $(i=1,4)$ [1] .

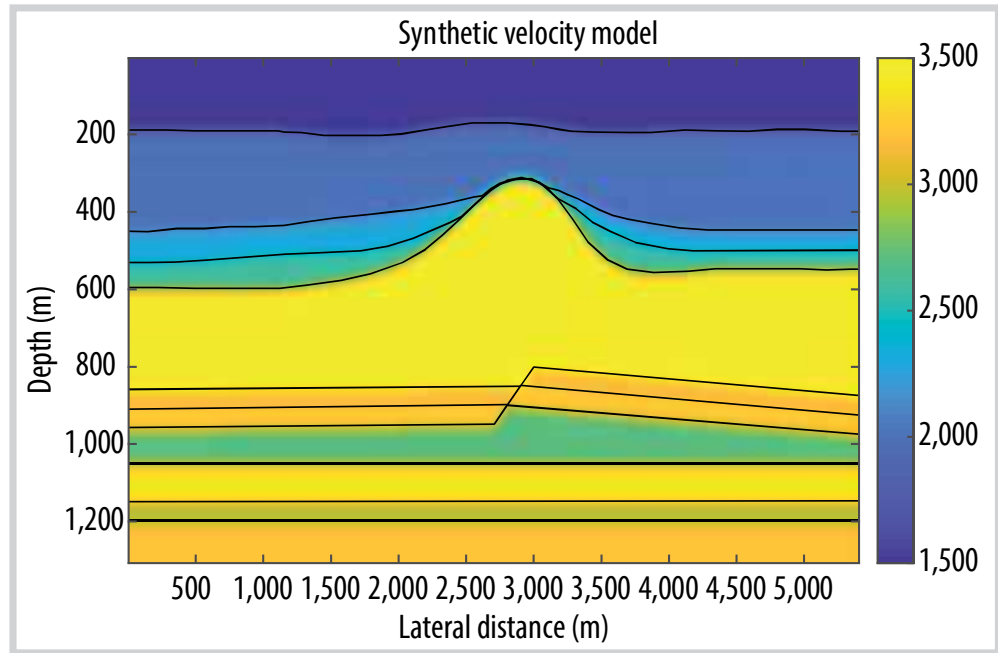

Figure 7. True velocity model with picked horizons (acoustic discontinuities).

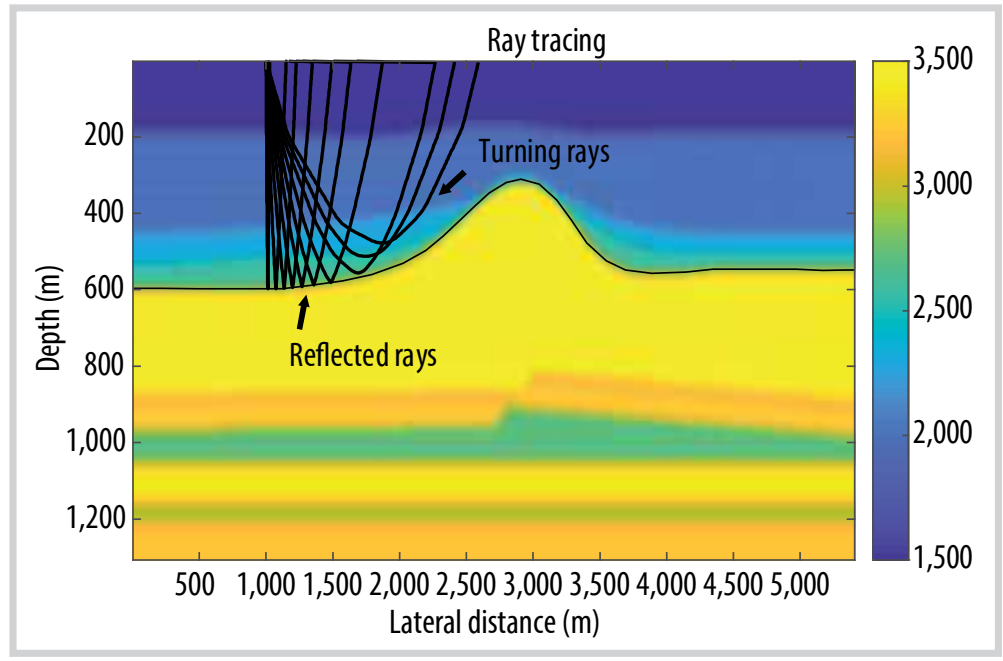

Figure 8. Ray tracing by Runge-Kutta method with a picked reflector.
To solve $S$ in Equation 10, normally the least-squares method is applied. However, a better way is to use the conjugate gradient (CG) method due to the nature of faster computational complexity. CG is the most popular iterative method for solving large systems of linear equations like Equation 9 because CG has a time complexity of $\mathrm{O}(m \sqrt{k})$, whereas the steepest descent has a time complexity of $\mathrm{O}(\mathrm{mk})$. Both algorithms have a space complexity of $O(m)$, where $k$ is the spectral condition number of matrix $D$, and $m$ is the number of non-zero entries in the matrix [5]. Upon application of a few CG iterations, the velocity model is updated to an optimised model much closer to the actual one.

\section{Synthetic data verification}

A synthetic velocity model (Figure 7) is used to verify the effectiveness of the method. The model is created in depth domain and contains ten seismic reflectors with an anticline in the high velocity region. There is also a fault-like structure in the deeper region of the model, which is an interesting object for the tomographic inversion study.

The optimisation procedure is applied to our true velocity model to estimate the shooting angle for each source-receiver pair and obtain the true travel time information, which is then used as the reference, or input, of iterative procedure.

The acquisition geometry was defined by a system composed of 207 sources (with a source interval of $25 \mathrm{~m}$ ) and 10 receivers for each source (with a receiver interval of $12.5 \mathrm{~m}$ ); all were placed on the water surface. Calculated travel times of all source-receiver pairs are used as reference data in tomographic inverse procedure. The inversion adopts the simple and powerful conjugate gradient method as mentioned in section 2.2. We use a strong smooth version of the true velocity model as the initial model for this step. The results of tomographic inversion test are presented in Figure 9. 


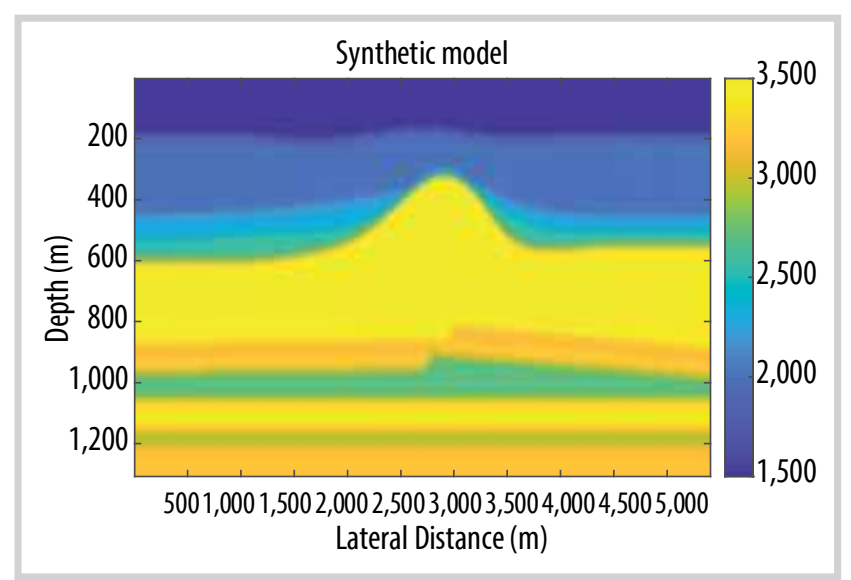

(a)

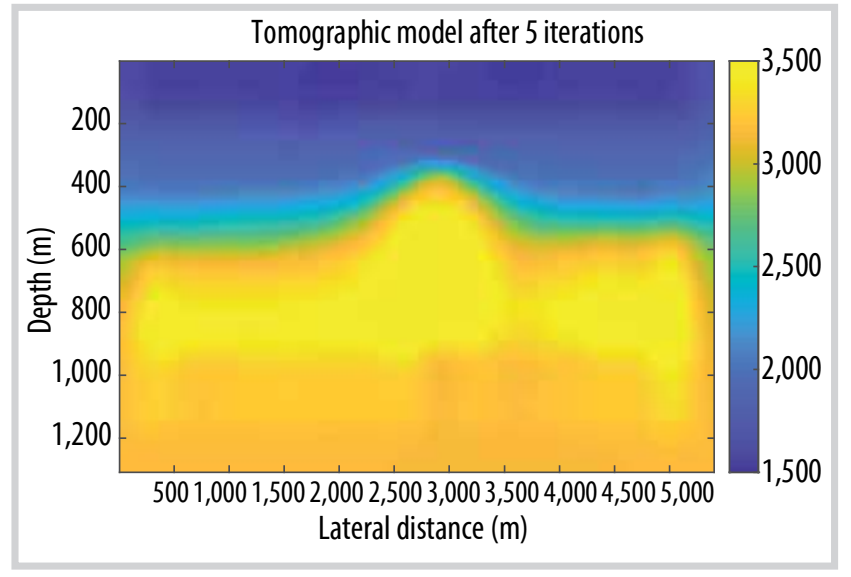

(c)

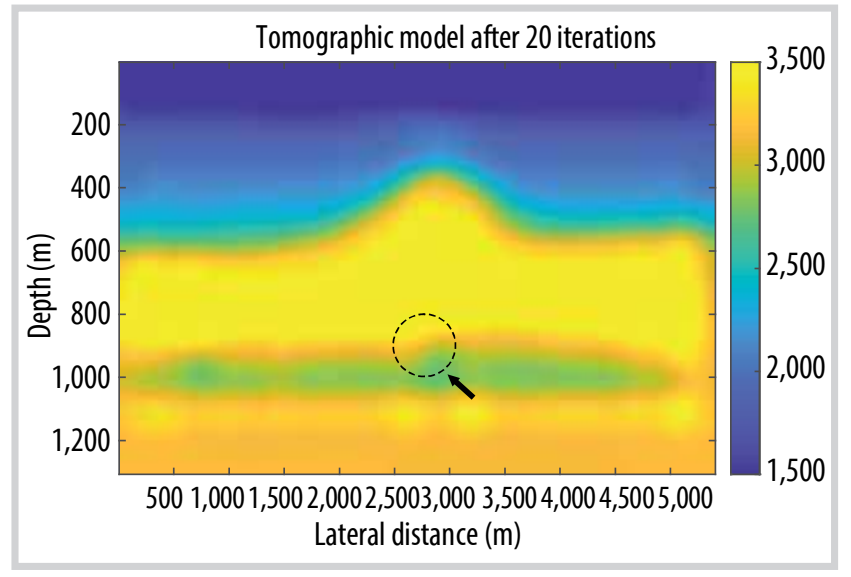

(e)

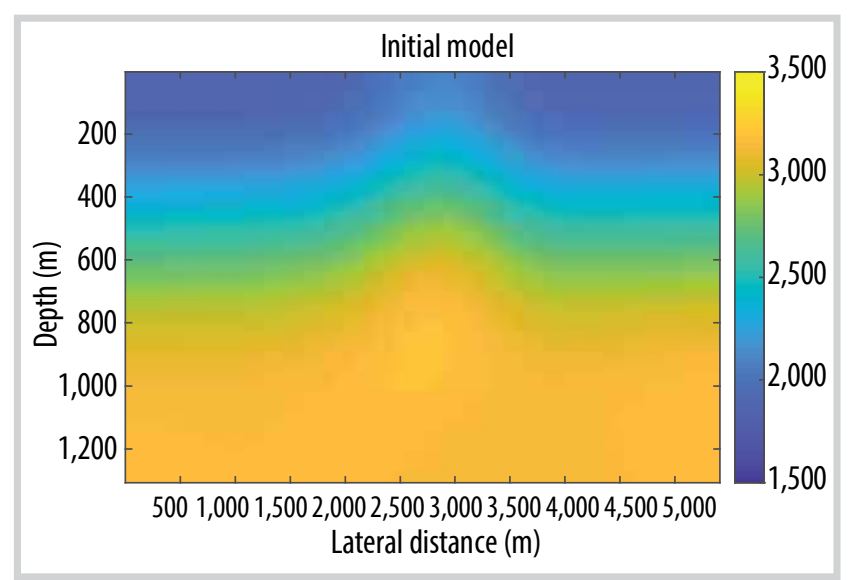

(b)

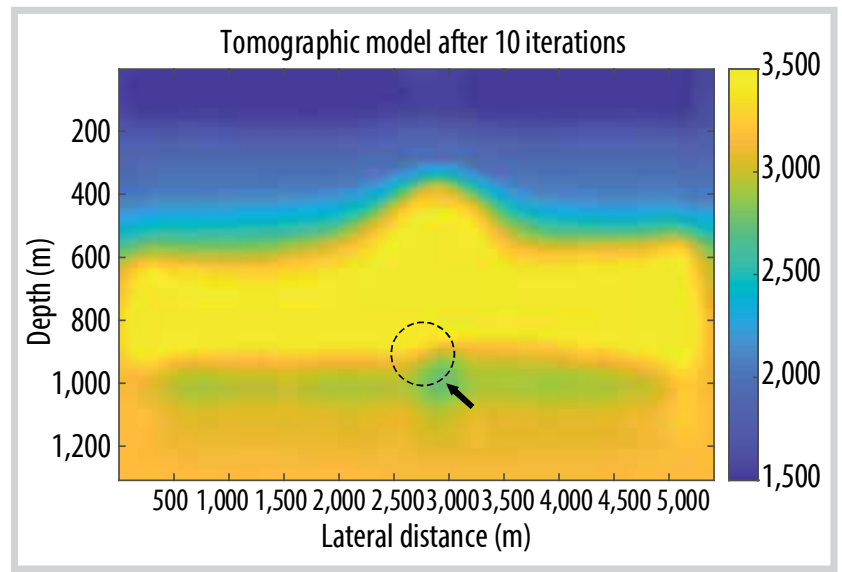

(d)

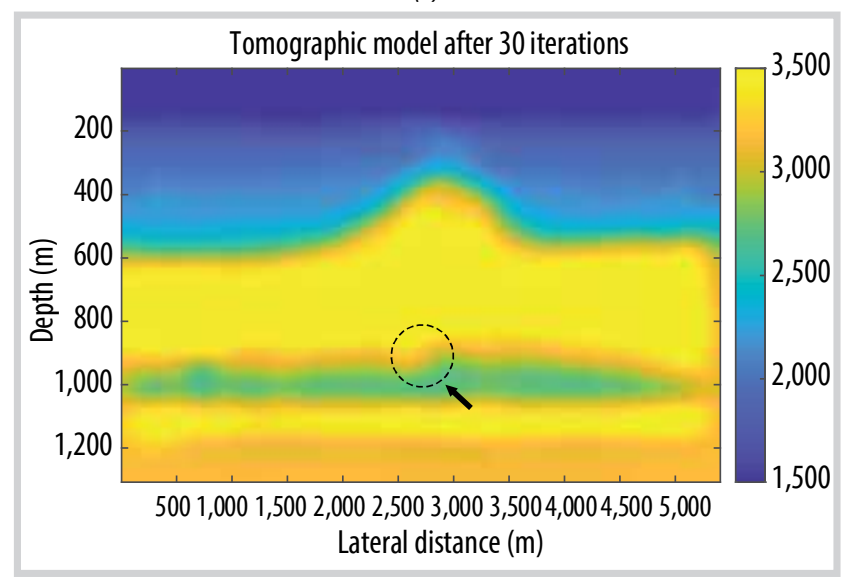

(f)

Figure 9. Results of tomographic inverse process. a) True velocity model; b) Initial model; c) Estimated tomographic model after 5 iterations; d) Estimated tomographic model after 10 iterations; e) Estimated tomographic model after 20 iterations; f) Estimated tomographic model after 30 iterations.

Table 3. Root-mean-square error of velocity models

\begin{tabular}{|c|c|c|}
\hline Model & RMSE (m/s) & Average relative error of velocity model (\%) \\
\hline Initial model & 370.6 & 18.15 \\
\hline Estimated model after 5 CG iterations & 219.6 & 10.75 \\
\hline Estimated model after 10 CG iterations & 176.4 & 8.65 \\
\hline Estimated model after 20 CG iterations & 156.1 & 7.65 \\
\hline Estimated model after 30 CG iterations & 152.9 & 7.5 \\
\hline
\end{tabular}




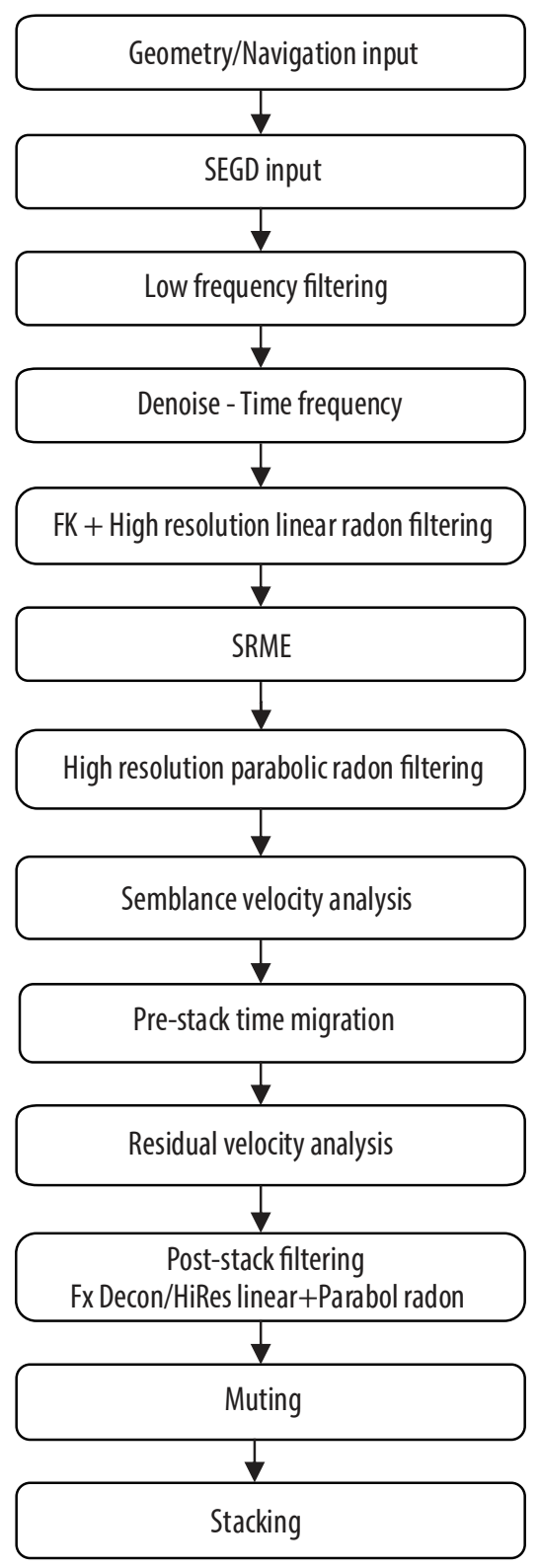

Figure 10. Basic processing steps.

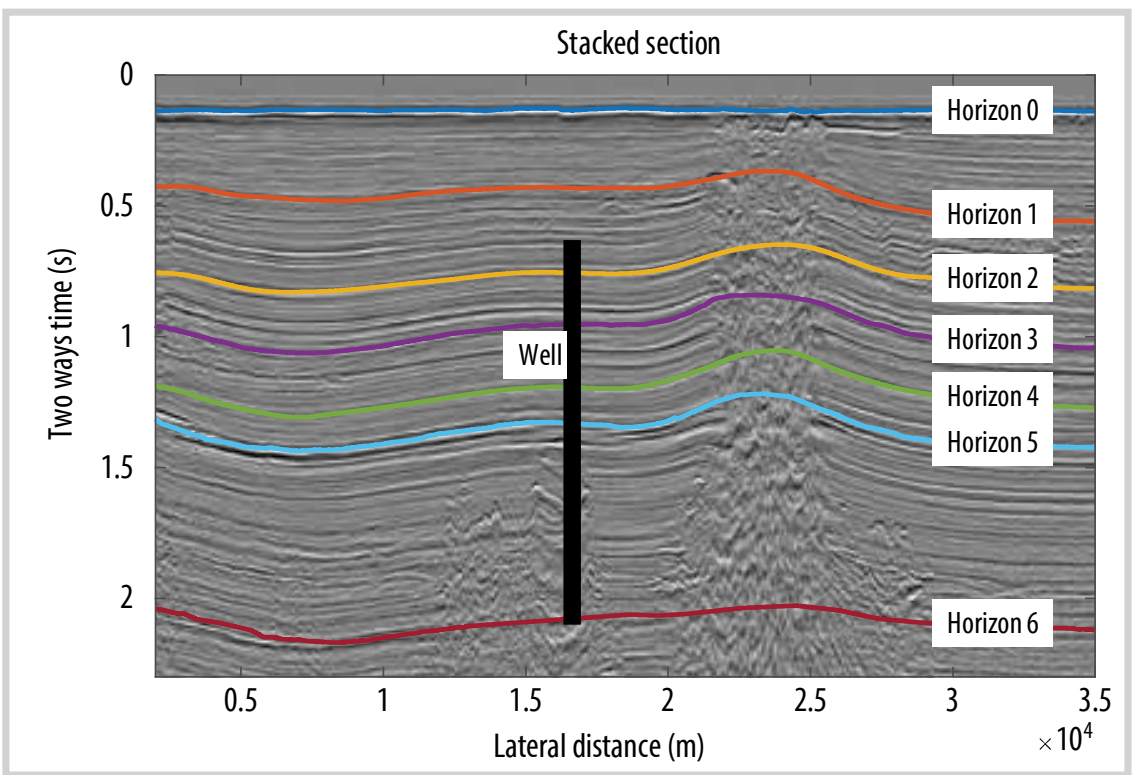

Figure 11. A seismic line used in the study with picked horizons and well location.

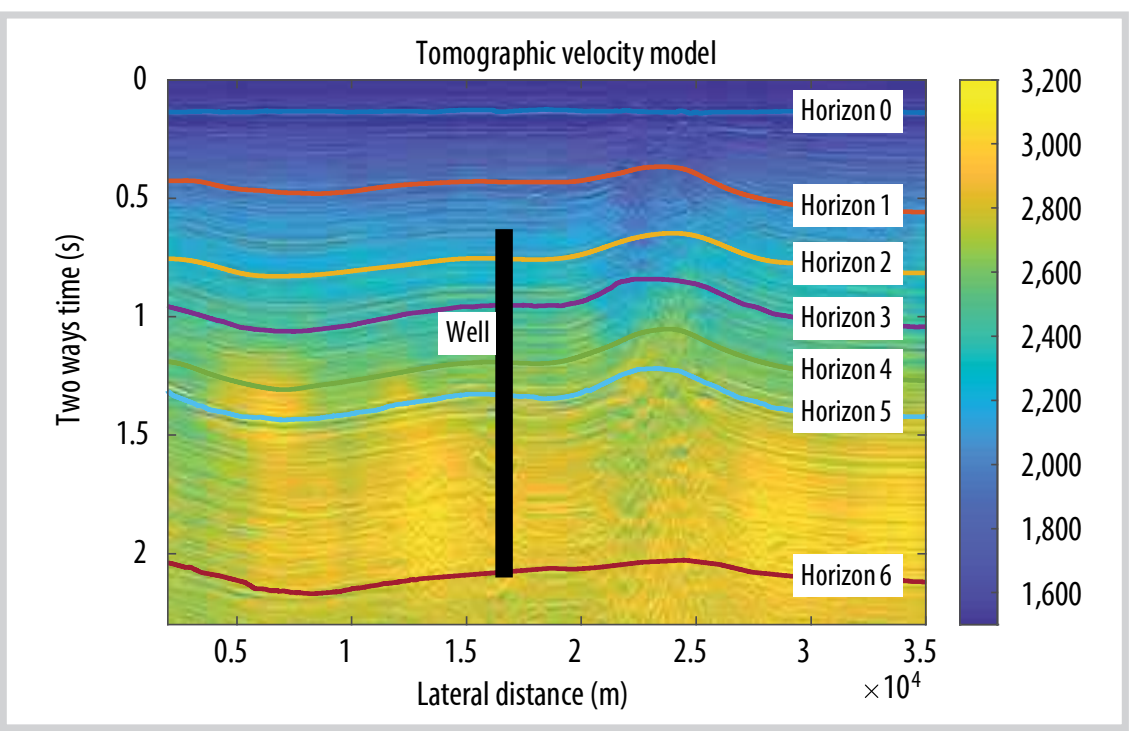

Figure 12. Tomographic velocity model overlaid with seismic data.
The algorithm starts from an over-smoothed model (Figure 9b) without any clear seismic features, and eventually converges to a much more realistic result. Shallow zone and high velocity section are recovered after only 5 CG iterations. By increasing the number of CG iterations, deeper layers of velocity model are also inverted more and more accurately. As seen in Figure $9 \mathrm{~d}$, the interesting fault-like structure is quickly recovered after 10 iterations. The algorithm accuracy can be estimated by calculating the root-mean-square error (RMSE). The smaller the RMSE, the closer the fit of estimated model to the true data. The RMSEs are shown in Table 3.

\section{Field data application}

We now present the application of our tomographic inverse algorithm to a 2D data set from a field offshore Vietnam. This data set is processed and interpreted by a geophysical team of the Vietnam Petroleum Institute (VPI). The basic processing steps are shown in Figure 10.

A distinct difference from the modelling case is that the data (velocity fields) in the synthetic modelling is already in the depth domain, while in the case of field application, the input data (seismic data, picked RMS velocity and picked horizons) is only in the time domain. Thus, there's a need to convert the input (picked horizons) from time to depth domain during the iterative procedure, which is 
not at a true depth but a depth depending on the current velocity model. By minimising the travel time residual, we hope the depths also converge to the true solution. Thus, beside the CG iterations to improve the velocity model to perform ray tracing with the current seismic-depthdomain horizons, there will be an outer loop to update the velocity model in depth with the updated depth of the horizons (Figure 1). Other than that, the inversion proceeds similarly to the synthetic modelling case.
Figure 11 shows stacked data with picked horizons and well location. From the process of applying algorithms to synthetic data we found that the more horizons are picked, the better the result of tomographic inversion will be. However, using more horizons also means more computational cost, especially for field data, where the number of shot-receiver pairs is usually a lot. In this work, we picked 7 horizons, among which 3 located above, 1

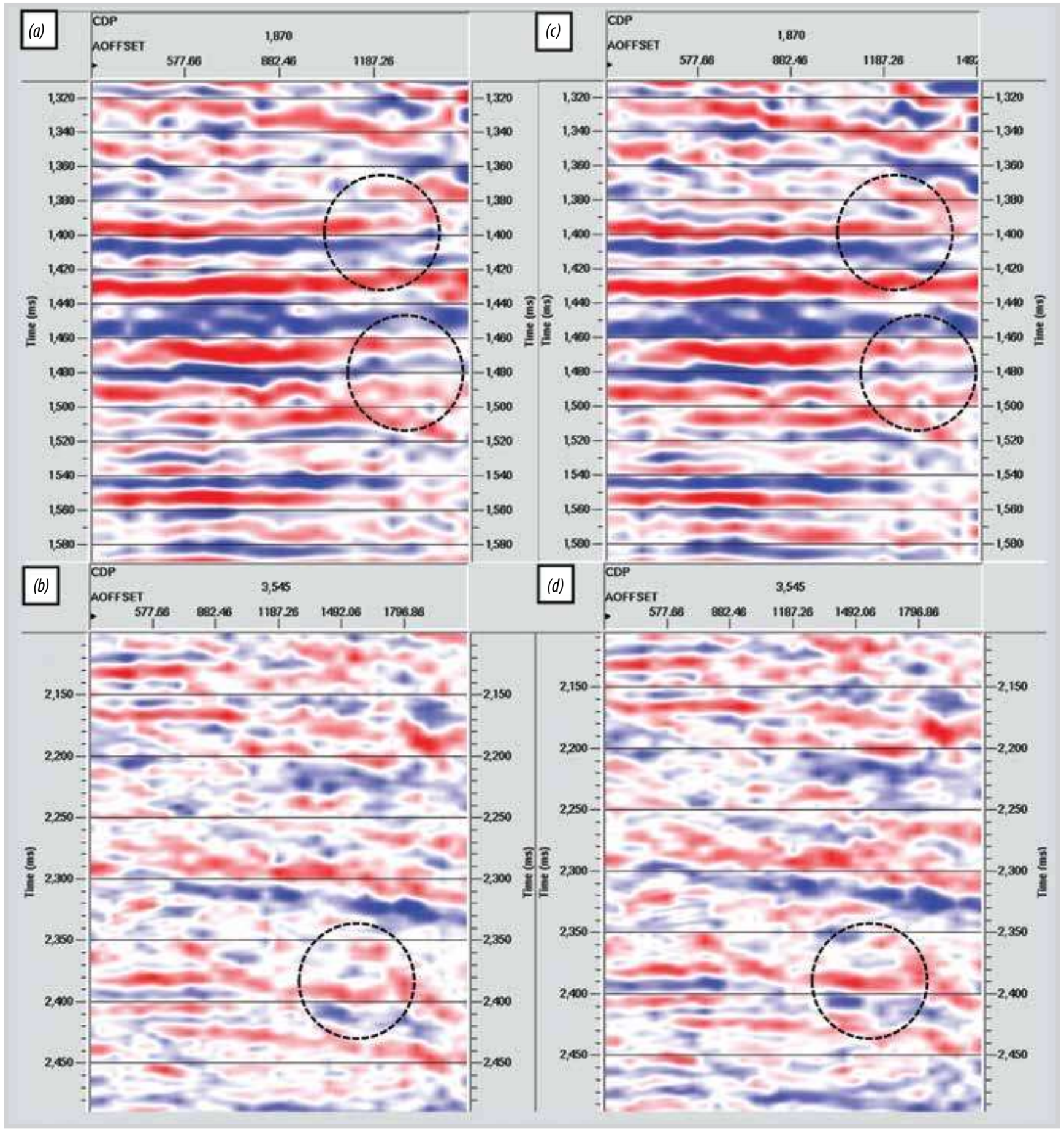

Figure 13. Comparison of CDP gatherings after PSTM with $(a, b)$ handpicked velocity and $(c, d)$ tomographic velocity. 
located below and 3 went through the region with well measurement data.

Once the velocity model is inverted by SeisT, it will be overlaid by stack section; then the velocity change as well as anomaly possibly associated with hydrocarbon accumulation can be observed as in Figure 12. In this particular seismic line, the seismic velocity model has been successfully inverted by the proposed SeisT algorithm, and the velocity surrounding the well location (at the diapir),

Table 4. Average residual of velocity models

\begin{tabular}{|l|c|}
\hline \multicolumn{1}{|c|}{ Model } & $\begin{array}{c}\text { Average travel time } \\
\text { residual (ms) }\end{array}$ \\
\hline Estimated model after 10 CG iterations & 3.664 \\
\hline Estimated model after 20 CG iterations & 3.147 \\
\hline Estimated model after 30 CG iterations & 3.093 \\
\hline Estimated model after 50 CG iterations & 3.033 \\
\hline
\end{tabular}

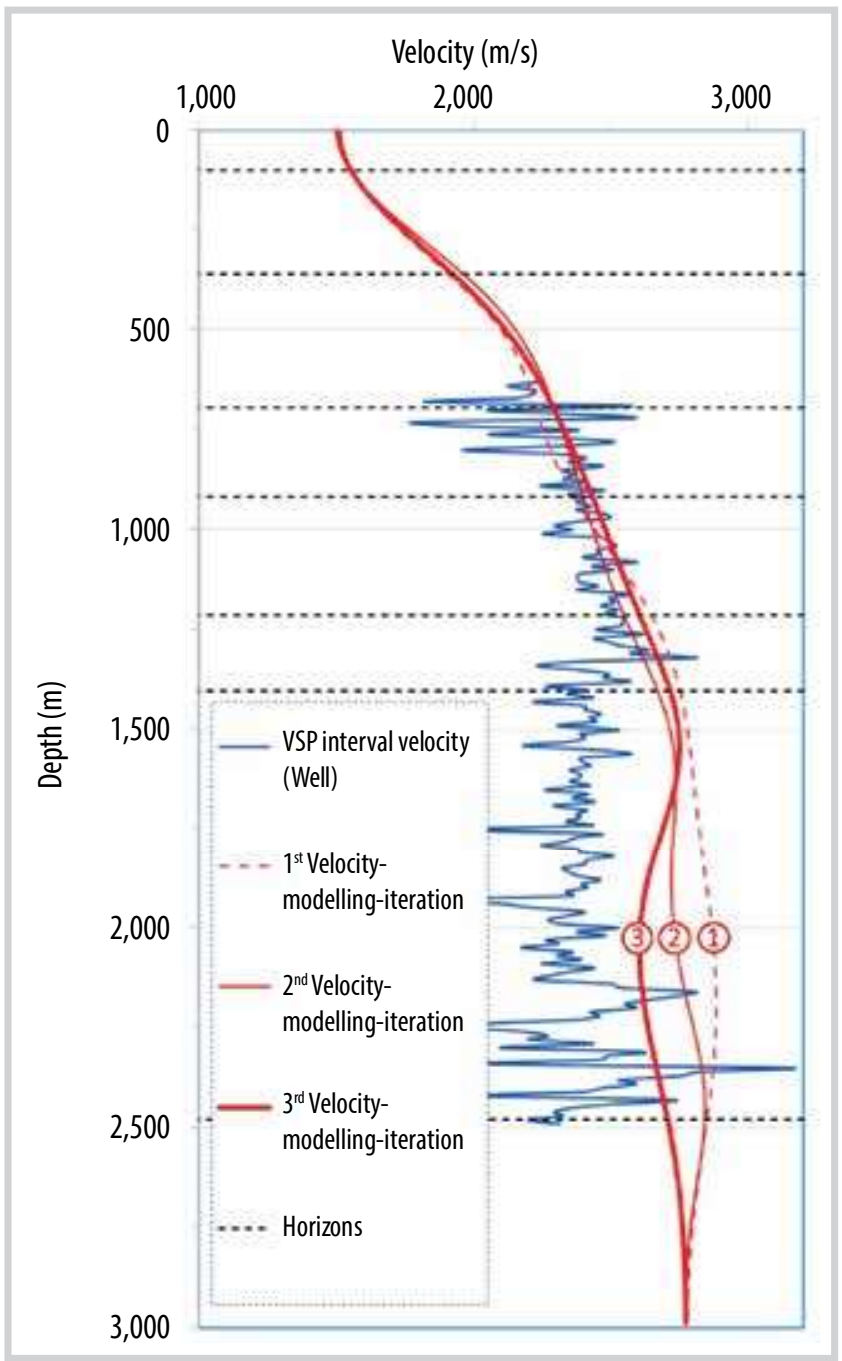

(a) where the gas was discovered, tends to be smaller than laterally surrounding area.

Unlike the case of synthetic model, for field data, we do not have an "absolutely" true velocity model to compare our tomographic results. So, we must try other ways to check if our algorithm works properly. In this work, we demonstrate two different methods for quality control (QC). One is using the estimated velocity model as the input for Kirchhoff Pre-Stack Time Migration (PSTM); its output is expected to contain flatten seismic events in the CDP gatherings (Figure 13). The other method is to compare the inverse model with the well data located nearby the studied line.

The average residuals of the estimated models are calculated as an additional verifying method. With the residual defined as the differences between the travel times estimated by the ray tracing method and the

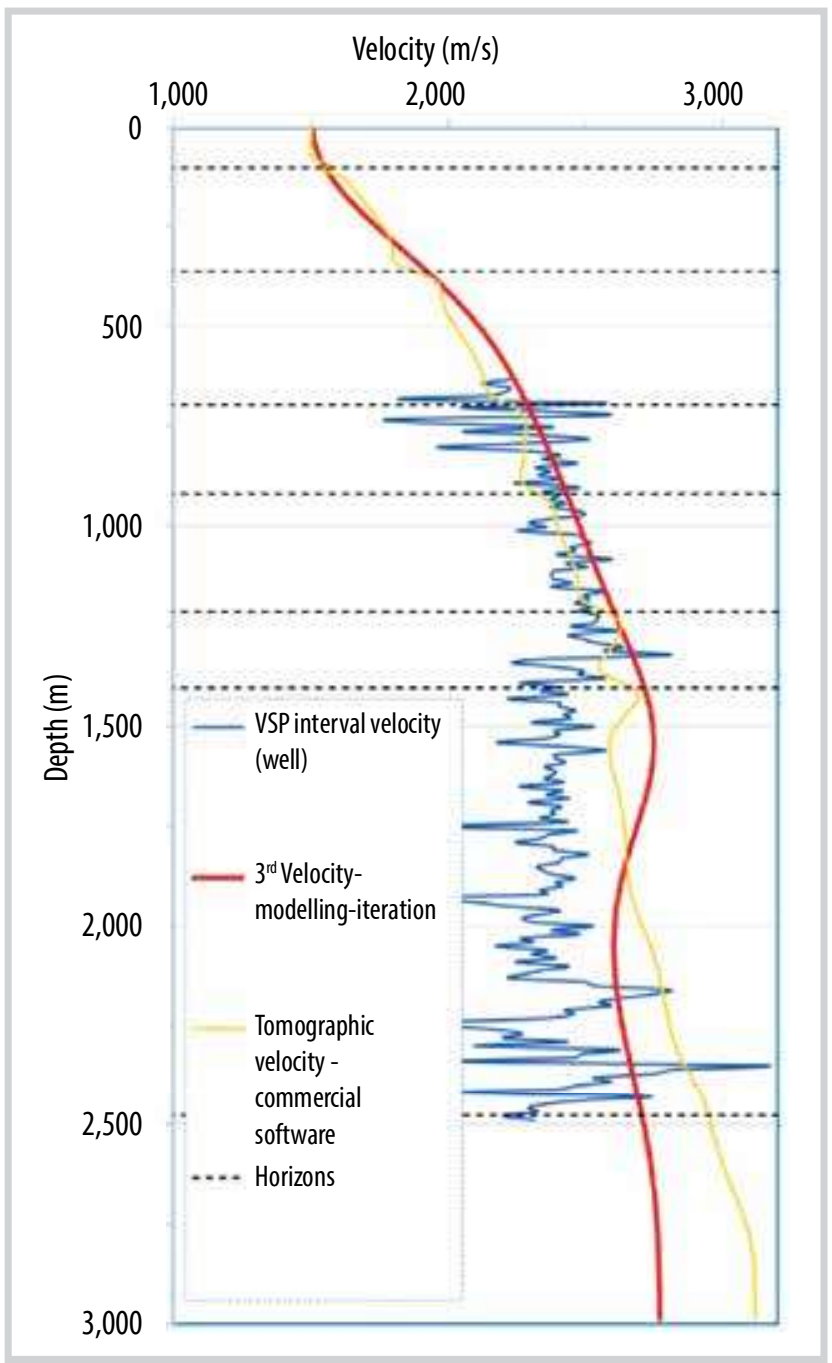

(b)

Figure 14. Comparison of tomographic velocity with (a) VSP velocity from well data and (b) commercial software. 
true travel times (horizons picked), the average residuals after CG iterations are calculated and shown in Table 4.

We can see from Table 4 that, when CG iteration increases, the average residual of the estimated model decreases more and more slowly. This phenomenon can be explained by the fact that, as the average residual gets closer to the sampling interval, it should be much harder for the optimisation routine to improve the results.

The location of picked horizons in the depth domain is calculated using a picked velocity model. This means if the velocity model is updated by tomographic inversion, the horizons' location should also be recalculated. For this reason, we have made several velocity modelling iterations, in each of which the location of the horizons is

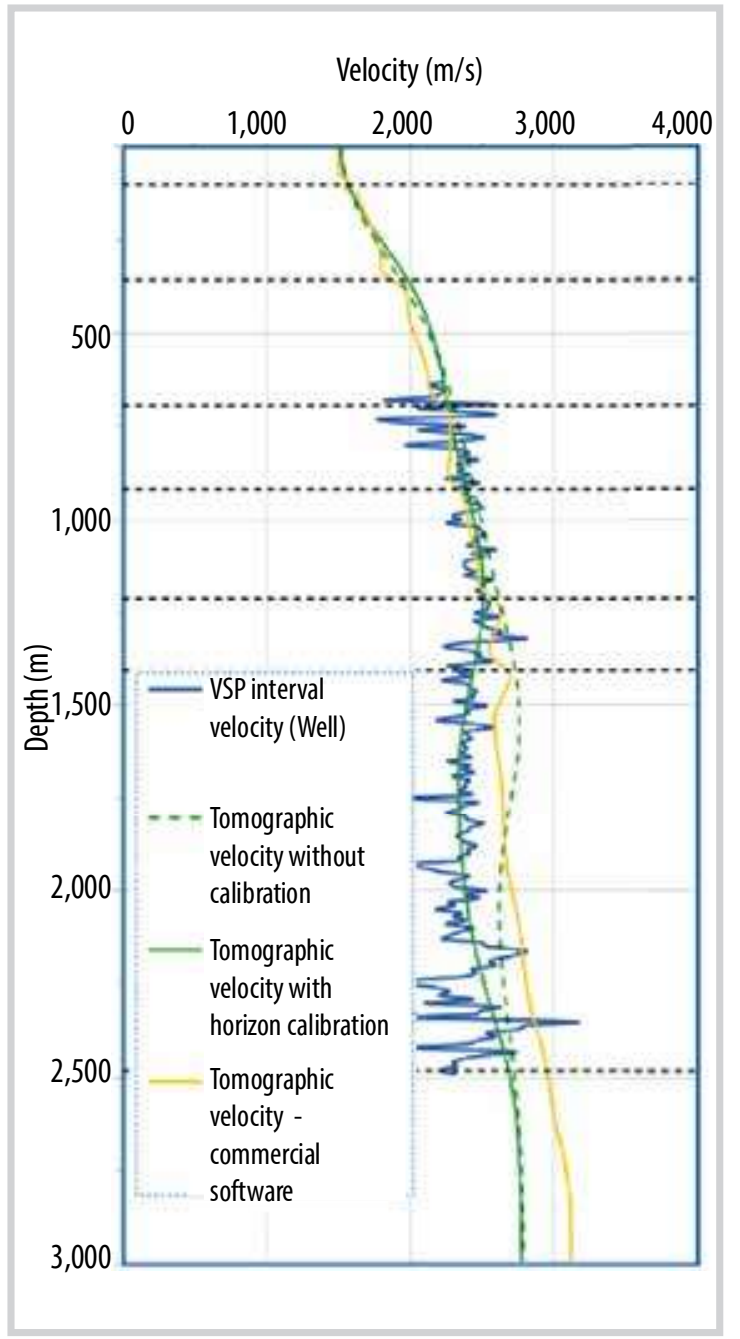

Figure 15. Comparison of tomographic velocity using well calibration with well data. defined using the velocity model estimated in the previous one. The improvement of this iterative procedure is shown in Figure $14 a$.

An independent check of the tomographic inversion result can be achieved by comparing to VSP velocity measurement of a local well (Figure 14); the well location is indicated in Figure 11. The tomographic inverted velocity is slightly higher than well VSP velocity, so the result is encouraging. A tomographic inversion result using a commercial software is also displayed in Figure 14b. The commercial software model (based on the grid-tomo algorithm) is better than ours in terms of frequency content - a room for our future improvements. However, our algorithm gives a better matched velocity estimation with the VSP velocity in the slow-velocity-anomaly zone (1,500 - 2,500 m).

We would also like to investigate the effect of "well calibration" as often performed in the industry. Effectively, the velocity model used to convert the horizons from time to depth domain has

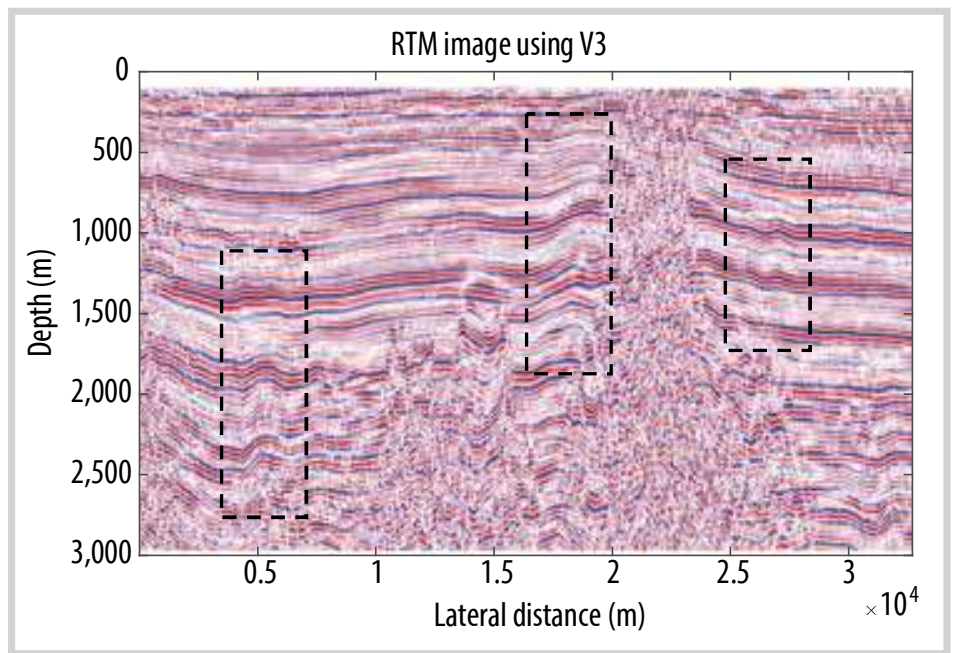

(a)

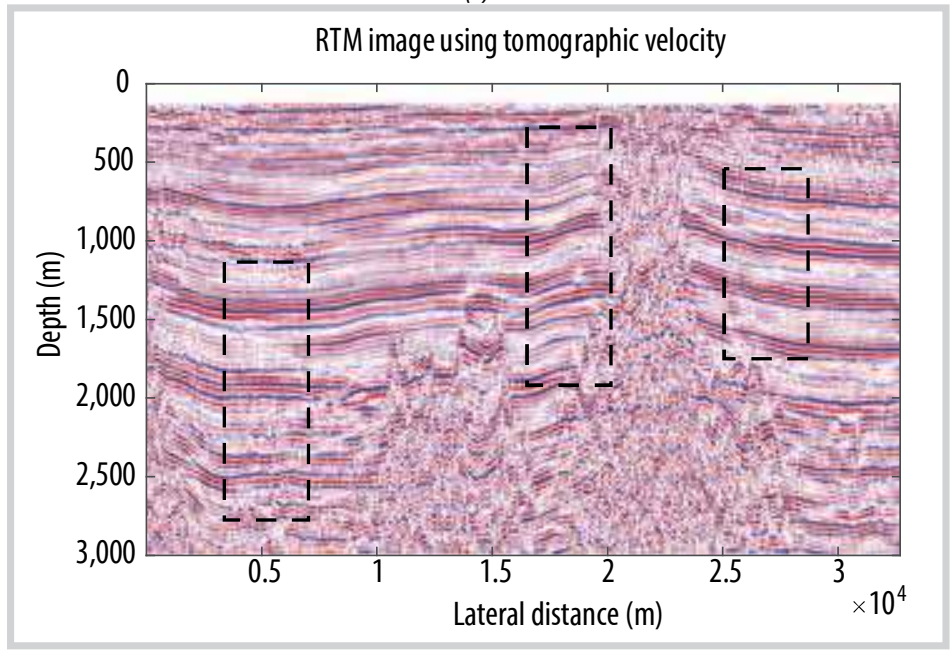

(b)

Figure 16. RTM images using (a) handpicked velocity V3 and (b) tomographic velocity. 
been calibrated to get closer to the well VSP velocity (at the well the model is matched with well data). The final tomographic inversion velocity at the well matches closely with the low frequency component (i.e. the trend) of the VSP velocity (Figure 15).

\section{Application of tomographic inversion results}

As an application of the tomographic velocity results, we used the Reverse Time Migration (RTM) to perform seismic depth imaging which can (hopefully) illustrate the improvements of the tomographic inversion compared to the handpicked velocity model. As shown in Figure 16b, seismic events in RTM image using tomographic velocity contain less artifacts than those using the handpicked velocity model (V3) in Figure 16a. The result in Figure 16b is partly comparable to the Kirchhoff migration result (Figure 11).

\section{Discussion}

The accuracy of horizon picking plays a significant role in ray-based reflection travel time tomography since the picked horizons will act as the reference arrival times for the inversion process. These horizons (after being converted to the depth domain) are also used in the ray tracing procedure, where arrival time of a particular source-receiver pair is estimated for the current version of the velocity model. This estimated arrival time is then compared to the reference one to optimise our velocity model. Thus, the quality of the ray-based reflection travel time tomography depends much on the quality of horizon picking, which is now performed manually. One way to improve the quality of horizon picking is to use auto-picking functions of software such as Kingdom, Landmark, and Petrel, etc. A more advanced auto-picking version can be obtained from PaleoScan software [6], which can simultaneously pick many horizons in a chronological order. Auto-picking can improve not only the quality but also the quantity of horizon picking in an efficiently short amount of time.

In the case where seismic reflectors are not evident such as in the zone of basement or salt/mud diapirs, seismic reflection travel time tomography might hit troubles. Therefore, a different approach must be considered, such as "common focus point (CFP) tomography" [7] or diffraction-based tomography [8].

During the implementation of the tomography algorithm, it is noted that, the system of equations in
(Equation 9) can only be solvable (in the least-squares sense) if the number of equations is greater than the number of unknowns. In general, the number of equations is equal to the number of source-receiver pairs times the number of horizons; and the number of unknowns equals to the number of grids in the velocity model. If the above condition is not satisfied (which is usually the case), additional constraints must be applied to solve this system of equations. A common constraint is using the smoothing filter to correlate nearby points. Other techniques such as constraint by "dip/azimuth" or "shaping regularisation" [9] can be considered too. Even with constraints, a non-uniqueness condition for the solution is possible and an accurate initial velocity model will be an important factor that strongly affects the quality of the final optimised model. An accurate initial model also greatly affects the speed of solving system (Equation 9) (less time to converge). For the field example, we use the smooth version of the hand-picked velocity (V3) as the initial model.

In general, the development of the reflection tomography technology by the VPI team has achieved several accomplishments:

- For the modelling data, the tomographic result has recovered some seismic characteristics in the original velocity model such as layering and fault features;

- In the field data application, the inverse tomographic velocity produces more flattened events on CDP gatherings than the hand-picked one after pre-stack migration;

- It can also be reliably used as the velocity model for the RTM module with improvement over artifacts, compared to the result using hand-picked velocity model.

However, there are still shortcomings such as the matching with the well data. The slight mismatch between the tomographic inversion and well VSP velocity could be attributed by (over-) smoothing of the velocity field, insufficient number of horizons, and/or the lack of modelling for other physical phenomenon modelling (anisotropy, Q effect...), which can be overcome partially by using the well calibration method.

\section{Conclusions}

In this work, the development of travel time reflection tomography technology at the VPI is demonstrated through synthetic and field results. Many issues faced by 
the team during the study and development of ray-based reflection travel time tomography have been discussed. We indicate that the quality of final tomographic results depends on many factors, especially the accuracy of the initial velocity model and the quality of horizon picking. We can reach further improvements by considering other constraint techniques (smoothing) and/or modelling more complicated physically, such as modelling $Q$ and/ or anisotropy effect. Although in this iteration of the technology, a basic tomographic inversion software and workflow are developed, its completion has highlighted some important insight of the process, including:

- The necessary algorithm, software module, and workflow for understanding the core techniques (ray tracing, optimisation, inversion) used in the tomographic inversion;

- The implementation of reflection tomography method to invert a better acoustic velocity model (compared to the normal handpick flow).

The basic model will need further development including more complicated physical models, such as $\mathrm{Q}$, anisotropic tomography. More advanced versions of velocity model building such as 3D tomography or full waveform inversion are also among the future goals.

\section{Acknowledgements}

We would like to thank the Vietnam Petroleum Institute (VPI) for the support given to the development of the travel time reflection tomography technology.

\section{References}

[1] Ian F. Jones, An introduction to: Velocity model building. EAGE, 2010.
[2] R.G. Pratt, F. Gao, C. Zelt, and A. Levander, "A comparison of Ray-Based and Waveform Tomography - Implications for migration", 64 $4^{\text {th }}$ EAGE Conference \& Exhibition, 2002. DOI: 0.3997/2214-4609-pdb.5.B023.

[3] Gary F. Margrave, "Raytracing for inhomogeneous media", Numerical Methods of Exploration Seismology with Algorithms in MATLAB. The University of Calgary, 2003, pp. $105-112$.

[4] Steven C. Chapra and Raymond P. Canale, Numerical methods for engineers. McGraw-Hill Education, 2015.

[5] Jonathan Richard Shewchuk, An introduction to the conjugate gradient method without the agonizing pain. School of Computer Science Carnegie Mellon University, 1994.

[6] Eliis, "PaleoScan software". [Online]. Available: https://www.eliis-geo.com/paleoscan-overview-a.html.

[7] B.E. Cox and D.J. Verschuur, "Tomographic inversion of focusing operators", 63 ${ }^{\text {rd }}$ EAGE Conference \& Exhibition, Netherlands, 11 - 15 June 2001. DOI: 10.3997/2214-4609-pdb.15.M-35.

[8] Mahasweta Mahapatra and Samiran Mahapatra, "Seismic diffraction tomography technique using very fast simulated annealing", Journal of Applied Geophysics, Vol. 67, No. 2, pp. 125 - 129, 2009. DOI: 10.1016/j. jappgeo.2008.10.004.

[9] Sergey Fomel, "Shaping regularization in geophysical estimation problems", Geophysics, Vol. 72, No. 2, 2007. DOI: 10.1190/1.2433716. 